\title{
Q fever in pregnant goats: humoral and cellular immune responses
}

Hendrik IJ Roest ${ }^{1 *}$, Jacob Post ${ }^{2}$, Betty van Gelderen ${ }^{1}$, Fred G van Zijderveld ${ }^{1}$ and Johanna MJ Rebel ${ }^{2}$

\begin{abstract}
Q fever is a zoonosis caused by the intracellular bacterium Coxiella burnetii. Both humoral and cellular immunity are important in the host defence against intracellular bacteria. Little is known about the immune response to C. burnetii infections in domestic ruminants even though these species are the major source of $\mathrm{Q}$ fever in humans. To investigate the goat's immune response we inoculated groups of pregnant goats via inhalation with a Dutch outbreak isolate of C. burnetii. All animals were successfully infected. Phase 1 and Phase 2 lgM- and IgG-specific antibodies were measured. Cellular immune responses were investigated by interferon-gamma, enzyme-linked immunosorbent spot test (IFN- $\gamma$ Elispot), lymphocyte proliferation test (LPT) and systemic cytokines. After two weeks post inoculation (wpi), a strong anti-C. burnetii Phase $2 \mathrm{lgM}$ and $\operatorname{lgG}$ antibody response was observed while the increase in IgM anti-Phase 1 antibodies was less pronounced. IgG anti-Phase 1 antibodies started to rise at 6 wpi. Cellular immune responses were observed after parturition. Our results demonstrated humoral and cellular immune responses to $C$. burnetii infection in pregnant goats. Cell-mediated immune responses did not differ enough to distinguish between Coxiella-infected and non-infected pregnant animals, whereas a strong-phase specific antibody response is detected after 2 wpi. This humoral immune response may be useful in the early detection of C. burnetii-infected pregnant goats.
\end{abstract}

\section{Introduction}

$\mathrm{Q}$ fever is a zoonosis caused by Coxiella burnetii. C. burnetii has a worldwide distribution except for New Zealand [1]. The bacterium has a wide host range including humans, terrestrial and marine mammals, birds and reptiles $[2,3]$. The zoonotic impact of the disease has recently been underlined by the Dutch Q fever outbreak in which 4029 human cases were registered during the years 2007-2010 [4,5]. More than 40000 people are assumed to be infected [6]. In this outbreak, C. burnetii-infected pregnant goats and sheep were the primary source of $\mathrm{Q}$ fever in humans $[7,8]$. During parturition these animals excrete high numbers of $C$. burnetii into the environment. Inhalation of C. burnetii-contaminated aerosols is the main route of infection in humans and can result in acute or chronic Q fever [9]. In the acute phase, humans suffer from a flu-like, self-limiting disease, atypical

\footnotetext{
*Correspondence: hendrikjan.roest@wur.nl

'Department of Bacteriology and TSEs, Central Veterinary Institute, part of Wageningen University and Research Centre, Edelhertweg 15, 8219, PH Lelystad, The Netherlands

Full list of author information is available at the end of the article
}

pneumonia or hepatitis. The chronic form of Q fever may lead to life-threatening endocarditis.

C. burnetii is a Gram-negative, intracellular bacterium. As in other Gram-negative bacteria (e.g. Brucella spp. and Enterobacteriaceae spp.), two major phenotypes (phases) of C. burnetii are recognised. Phase 1 of C. burnetii corresponds with the smooth phase of other Gram-negative bacteria and expresses full-length lipopolysaccharide (LPS) on its surface. Phase 2 corresponds with the rough phase of Gram-negative bacteria and lacks the O-antigenic region on its LPS [10]. Phase 1 is highly virulent and able to replicate in natural hosts, while Phase 2 Coxiella are considered avirulent and unable to replicate in immunocompetent animals $[11,12]$. The phase variation is interesting for the humoral immune response after Coxiella infection in mice, guinea pigs and humans. Following the inoculation of mice and guinea pigs with $C$. burnetii Phase 1 , antibodies that recognise both Phase 1 and Phase 2 C. burnetii are generated $[11,12]$. In humans, the detection of phase-specific antibodies plays an important role in the diagnosis of acute and chronic $Q$ fever [13]. This has not been investigated yet in goats, but is of importance specially in
C Biomed Central

(c) 2013 Roest et al.; licensee BioMed Central Ltd. This is an Open Access article distributed under the terms of the Creative Commons Attribution License (http://creativecommons.org/licenses/by/2.0), which permits unrestricted use, distribution, and reproduction in any medium, provided the original work is properly cited. 
pregnant goats as these risk animals for human $\mathrm{Q}$ fever do not excrete $C$. burnetii during pregnancy and can therefore not be detected via the excretion of the bacterium [14]. Tools might be found that can help in the early diagnosis of $\mathrm{Q}$ fever in (pregnant) goats and can provide insights into the herd dynamics of $\mathrm{Q}$ fever infections, similar to those already anticipated in cattle herds [15].

The role of cellular immunity in the host defence against $C$. burnetii infections is not well established. In mice it is suggested that T cells are particularly important for the clearance of the bacterium after infection. Interferon-gamma (IFN- $\gamma$ ) and tumour necrosis factoralpha (TNF- $\alpha$ ) seem to be essential for the early control of Coxiella proliferation [12]. Furthermore, in vitro studies with human, peripheral blood, mononuclear cells indicate specific stimulation of $\mathrm{T}$ cells by human, monocytederived, dendritic cells (HMDCs) pulsed with C. burnetii outer membrane protein Com1 [16]. Recently the value of the interferon-gamma, enzyme-linked, immunosorbent spot test, a diagnostic tests based on cellular immunity, in the diagnosis of chronic Q fever in humans has also been shown [17]. In pre-vaccination screening of humans, a skin test is used to detect previously sensitised people so as to avoid adverse reactions after $\mathrm{Q}$ fever vaccination in these persons [18]. In cattle, a skin test with a diluted vaccine antigen has been suggested as a read out for a cellular response in an attempt to assess the duration of immunity after Q fever vaccination [19]. Cellular immune responses after $C$. burnetii infection of domestic ruminants have not been investigated, although this may provide potential tools to investigate the pathogenesis of $C$. burnetii infection in ruminants and to improve its diagnosis. In addition, the vaccine efficacy in already infected hosts can probably be improved if the cell-mediated immunity can be stimulated with a new generation of vaccines.

The goal of the present study was to investigate the humoral and cellular immune response in pregnant goats after inoculation with the Dutch outbreak strain of C. burnetii. The humoral immune response was measured by detecting anti-Phase 1 and anti-Phase 2 IgM- and IgG-specific antibodies. Cellular immune responses were measured with the interferon-gamma, enzyme-linked, immunosorbent spot test (IFN- $\gamma$ Elispot) and the lymphocyte proliferation test (LPT) as well as by measuring systemic mRNA for different cytokines. Our results indicate a strong phase-specific IgM and IgG antibody response during early infection, whereas the cell- mediated immune response did not differ much between the Coxiella-infected pregnant goats and the non-infected animals.

\section{Materials and methods Inoculum}

C. burnetii strain X09003262-001 was isolated from a placenta of a dairy goat that aborted on a farm during the $\mathrm{Q}$ fever outbreak in the Netherlands. This has previously been described [14]. In short, part of an immunohistochemically confirmed C. burnetii-positive placenta was crushed and filtered before inoculation with a culture of Buffalo Green Monkey (BGM) cells kept in culture medium without antibiotics (EMEM with $10 \%$ bovine serum albumin, 1\% NEAA, 1\% glutamax). The inoculated cells were incubated for 14 days at $37^{\circ} \mathrm{C}$ in a closed flask and culture medium was refreshed twice a week. The cell culture was negative for Chlamydia abortus, Simkania negevensis and mycoplasma. A large batch of strain X09003262-001 was prepared and the mouse-infective dose (MID) of the batch was determined. Prior to inoculation, the inoculum was adjusted to the required MID by dilution with culture medium. The strain was genotyped as CbNL01, the predominant C. burnetii genotype in the Dutch $Q$ fever outbreak. To ensure inoculation of Phase 1 bacteria, cell culture passage 2 of the field isolate was used. In the inoculum, no Phase 2 C. burnetii were detected with an immunofluorescence test set up with the serum of a goat with a high antiPhase 2 antibody titre but no Phase 1 titre. All experiments were approved by the Animal Experiment Commission of the Central Veterinary Institute, part of Wageningen UR, in accordance with Dutch regulations on animal experimentation.

\section{Animal experiment}

The experimental set up has previously been described (Experiment III, [14]). Sixteen healthy, pregnant, serologically $Q$ fever negative, Alpine yearling goats were purchased from INRA (Institut National de la Recherche Agronomique, Domaine de Galle), France. Pregnancy and the duration of pregnancy were confirmed using ultrasound. All goats tested serologically negative for antibodies against C. burnetii and Chlamydia abortus on the day of arrival. Six negative control goats were housed in animal biosafety level (aBSL) 2 facilities. Two groups of 5 goats (Group A and B) were separately housed in aBSL3 facilities for inoculation with $C$. burnetii. On day 76 of pregnancy, 10 goats were intranasally inoculated with $1 \mathrm{~mL}$ containing $10^{6}$ MID C. burnetii while the six negative control animals were intranasally inoculated with $1 \mathrm{~mL}$ of culture medium. General health was monitored by daily clinical inspection of behaviour, appetite and consistency of the faeces. All goats were kept alive until the end of the experiment at 13 weeks post inoculation (wpi) (negative controls and Group A) and 14 wpi (Group B). Weak-born kids were euthanised when necessary for ethical reasons and liveborn kids were kept together with their does until the end of the experiment.

During the experiment goats did not show any clinical signs of disease except for abortion. Three of the ten Coxiella-inoculated goats aborted (one single kid and 
two twins) at 7, 8 and 10 wpi respectively. One goat delivered one stillborn kid and one liveborn weak kid at 10 wpi. Two goats delivered one stillborn and one liveborn healthy kid each at 9 wpi; one goat delivered two weak kids at 9 wpi and three goats delivered healthy kids, all singles at 9 wpi. The six control goats delivered healthy liveborn kids at 10 and 11 wpi, which was equal to 149 to 157 days of gestation. C. burnetii was detected in the placentas of all ten Coxiella-inoculated goats by immunohistochemistry and by PCR in the vaginal mucus just after parturition. These results indicate that all Coxiella-inoculated goats were successfully infected with C. burnetii.

\section{Sampling}

Jugular blood was sampled from each of the goats weekly from 0 wpi just before the inoculation until $13 \mathrm{wpi}$, except that at 4 wpi goats were not sampled and at $13 \mathrm{wpi}$ the negative control goats were not sampled for antibody detection for logistical reasons. When results from samples were related to parturition, parturition was set at time point 0 ; results from samples taken in the week before parturition were indicated as week -1 and results from samples taken in the week after parturition were indicated as week 1 . Blood was collected in coagulation tubes for antibody detection, in anticoagulation tubes (EDTA) for cell-mediated immunity and in PAXgene ${ }^{\circledR}$ Blood RNA tubes to preserve cytokine's mRNA.

Detection of phase 1 and phase $2 \lg M$ and $\lg G$ antibodies C. burnetii Phase 1 and Phase 2 IgM- and IgG-specific antibodies were detected in an ELISA format. C. burnetii Phase 1 and Phase 2 ELISA-specific plates were purchased from Virion/Serion (Serion ELISA classic Coxiella burnetii Phase 1 and Phase 2, Germany). Optimal serum and conjugate dilutions were determined in advance and positive and negative controls were selected (data not shown). Plates were incubated with $100 \mu \mathrm{L}$ 1:160 diluted serum in phosphate buffered saline (PBS), $\mathrm{pH} 7.2$ with $0.5 \mathrm{ml} 10 \%(\mathrm{v} / \mathrm{v})$ tween $80(\mathrm{PBS}-\mathrm{Tw})$ for $1 \mathrm{~h}$ at $37^{\circ} \mathrm{C}$. After incubation, plates were washed automatically (Schleicher, Germany), 6 times with $1400 \mu \mathrm{L}$ of $0.5 \%$ Tween 20 in water and incubated for $1 \mathrm{~h}$ at $37^{\circ} \mathrm{C}$ with $100 \mu \mathrm{L}$ of diluted alkaline phosphatase-conjugated antibodies. For the detection of IgM antibodies rabbit antigoat IgM (Bioconnect, the Netherlands) antibodies were used, 1:1000 diluted in PBS-Tw and $0.5 \mathrm{M} \mathrm{NaCl}$ for the detection of Phase 1 antibodies or 1:5000 diluted for the detection of Phase 2 antibodies. For the detection of IgG antibodies rabbit $\mathrm{F}\left(\mathrm{ab}^{\prime}\right) 2$ anti-goat IgG (H/L) (Bioconnect, the Netherlands) were used, 1:2000 diluted for the detection of Phase 1 antibodies or 1:4000 diluted for the detection of Phase 2 antibodies. After incubation with the conjugate, plates were washed as described above and $100 \mu \mathrm{L}$ of para-nitrophenylphosphate substrate (Virion/Serion, Germany) per well was added and the reaction was stopped after $30 \mathrm{~min}$ at $37^{\circ} \mathrm{C}$ with $100 \mu \mathrm{L}$ of $1.2 \mathrm{~N}$ sodium hydroxide (Virion/Serion, Germany). The optical density (OD) was measured at $405 \mathrm{~nm}$ (EL 808 Ultra microplate reader, Bio-tek instruments, USA). On each plate the same negative and positive control serum was tested in duplicate per phase/Ig combination. Results of the serum were given related to the average positive control OD, both corrected for the average negative control OD.

\section{Interferon-gamma, enzyme-linked, immunosorbent spot test (IFN- $\gamma$ Elispot)}

Peripheral blood mononuclear cells (PBMCs) were isolated from EDTA blood by Ficoll-Hypaque (Amersham Biosciences, Sweden) density gradient centrifugation. Interferongamma, enzyme-linked immunosorbent spot test (IFN- $\gamma$ Elispot) assay was performed using $5 \times 10^{5}$ PBMCs per well (MSIPS4W10 plates, Millipore, USA) from each goat. Detection of the T cell-produced IFN- $\gamma$ was performed with the Elispot kit for Bovine/Ovine/Equine IFN- $\gamma$ (MabTech, Nacka Strand, Sweden). We evaluated the kit for caprine IFN- $\gamma$ and found it suitable for use (data not shown). $C$. burnetii $\mathrm{T}$-cell responses were examined after stimulation with culture medium (negative control), C. burnetii strain Nine Mile Phase 1 and Phase 2 (Virion/Serion 1227, 1:5000 diluted in culture medium) or ConA (positive control). IFN- $\gamma$ spot-forming cells were counted using an ImmunoSpot analyzer (CTL, USA). We optimised counting parameters to precisely and accurately count all Elispot plates. The average results of all goats in the group are given as relative results of the positive control after correction for the medium control.

\section{Lymphocyte proliferation test (LPT)}

PBMCs suspended in medium (negative control), C. burnetii strain Nine Mile Phase 1 and Phase 2 (Virion/Serion 1227, 1:5000 diluted in culture medium) or ConA (positive control) were added to triplicate wells. Plates were incubated at $37^{\circ} \mathrm{C}$ for $72 \mathrm{~h}$. For the last $18 \mathrm{~h}$ of incubation, alamar blue (Invitrogen, USA) was added. Supernatant was harvested and absorbance was measured at $570 \mathrm{~nm} / 600 \mathrm{~nm}$. Results are given as average results per group. Individual results were calculated as relative induction of the average difference of the OD at $600 \mathrm{~nm}$ and $570 \mathrm{~nm}$ of the sample in duplicate compared to the medium control.

\section{Cytokine mRNA induction}

Blood specimens $(2.5 \mathrm{~mL})$ collected in PAXgene ${ }^{\circledR}$ tubes were incubated at room temperature for $4 \mathrm{~h}$ for RNA stabilisation and then stored at $-80^{\circ} \mathrm{C}$. RNA was extracted from whole blood using the manufacturer's guidelines. In 
brief, samples were removed from $-80^{\circ} \mathrm{C}$ and incubated over night at $4^{\circ} \mathrm{C}$ to ensure complete lysis of the blood cells. Then tubes were centrifuged for $10 \mathrm{~min}$ at $4000 \mathrm{~g}$, the supernatant was discarded and $5 \mathrm{~mL}$ of RNase-free water was added to the pellet. The pellet was resuspended. Washing was repeated and the pellet was finally resuspended in $1 \mathrm{~mL}$ trizol (Invitrogen, USA). Subsequently, a phase separation with chloroform was performed and RNA was precipitated using 2-propanol. Additional purification was performed with the DNA-free kit (Ambion, USA). The quality and integrity of the RNA samples were analysed using the Agilent Bioanalyzer (lab on chip, Agilent Technologies, USA). For the quantification of cytokine mRNA, cDNA was made using random hexamer primers and reverse transcriptase. Forward and reverse primers were selected to detect the CDNA of TNF- $\alpha$, IL$1 \beta$, IFN- $\alpha$, IFN- $\gamma$, IL-2 and IL-10 (Table 1). PCR was performed using Syber Green PCR Master Mix (Applied Biosystems, USA) in an ABI 7500 Real-Time PCR system (PE Applied Biosystems, USA). Results were quantified and normalised compared to the amount of succinate dehydrogenase complex subunit A gene (SDHA) of the same sample. SDHA was selected as reference gene on basis of the geNORM Bos Taurus housekeeping gene selection kit (Primerdesign, UK) as the best performing housekeeping gene compared to the other housekeeping genes included in the kit. For the quantification, a standard curve of the plasmid with the insert of the cytokine of interest constructed in pGEM-T easy (Promega, the Netherlands) was used. For the determination of the amount of the reference gene per sample, a standard curve of mRNA SDHA in water was used. For negative controls, RNA samples without reverse transcriptase in the reaction mixture were used.

Table 1 Sequences of the forward en reverse primers used to detect the DNA transcripts of the mRNA of goat's TNF- $\alpha$, IL-1 $\beta$, IFN- $\alpha$, IFN- $\boldsymbol{\gamma}$, IL-2 and IL-10

\begin{tabular}{llll}
\hline Gene of interest & NCBI code & Primer & Sequence \\
\hline INF- $a$ & X14828 & Forward & CCTTGAGAGATCTCACCTA \\
\hline IL-1 $\beta$ & & Reverse & CAAACATAAACAGAGGGAGT \\
\hline & DQ837160 & Forward & TACCTGTCTTGTGTGAAAAA \\
\hline IFN-a & Reverse & CAAATTCAACTGTGTTCTTG \\
\hline IFN- $\gamma$ & FJ959074 & Forward & GAGGAAATACTTCCACAGAG \\
\hline & & Reverse & ATGACTTCTGCTCTGACAAC \\
\hline IL-2 & EF375708 & Forward & GAAATTTGAAGAATTGAA \\
\hline & Reverse & AATGACCTGGTTATCTTTGA \\
\hline IL-10 & & Reverse & ACATCCAAATGAGTTCTGTT \\
\hline & DQ837159 & Forward & GGCAAAGTGAAGACTTTCT \\
\hline
\end{tabular}

NCBI National Center for Biotechnology Information.

\section{Statistical analyses}

A two-way analysis of variance (ANOVA) with infection status (i.e. infected with $C$. burnetii and non-infected goats) and sample week as independent variables and the results of the used tests (i.e. phase specific ELISAs, IFN- $\gamma$ Elispot, LPT and the different cytokines) as dependent variable was used to compare the difference between de parameters of the two groups of animals (IBM SPSS Statistics 19). Homogeneity of variances was preliminary tested using Levene's test. $P$ values $\leq 0.05$ correspond with a confidence interval of $95 \%$, marked as "; $P \leq 0.01$ correspond with a confidence interval of $99 \%$, marked as $*$.

\section{Results}

\section{Humoral immune response}

Ten pregnant goats were intranasally inoculated with a Dutch C. burnetii outbreak strain and jugular blood was sampled weekly. The serum of each goat was tested for IgM and IgG $C$. burnetii-specific antibodies using $C$. burnetii Phase 1- and Phase 2-specific ELISAs. Average IgM antibody levels against Phase 2 antigen (IgMph2) rised significantly from three weeks post-inoculation (wpi) onwards; after 3 wpi the antibody titre decreased and stabilised until the end of the experiment (Figure 1a). Average anti-C. burnetii Phase 2 IgG antibody levels (IgGph2) rapidly increased between 2 wpi and 4 wpi and then at a slower rate till 10 wpi (Figure 1b). Average anti-C. burnetii Phase 1 IgM antibody levels (IgMph1) started to rise from 3 wpi onwards and a significant difference between the infected and non-infected group was present at 4 wpi and from 8 wpi till the end of the experiment (Figure 1c). AntiC. burnetii Phase 1 IgG antibody levels (IgGph1) started to rise at 6 wpi; at 9 wpi the average titre stabilised until the end of the experiment at 12 wpi (Figure 1d). IgMph2 and IgGph2 levels were not influenced by parturition. The second rise of IgMph1 started 1 week before parturition. IgGph1 started to rise 4 weeks before parturition. The data indicate a strong IgMph2 and IgGph2 response starting after two weeks after inoculation. The IgMph1 and IgGph1 response started later after inoculation.

\section{Cell-mediated immune response}

To investigate the cell-mediated immune response, IFN- $\gamma$ Elispot and the LPT were performed on cells isolated from the jugular blood samples. In these tests C. burnetii Phase 1 and Phase 2 antigens were used as stimulus. In the first four weeks after inoculation, no significant difference between the Coxiella-inoculated goats and the control goats was observed in the IFN- $\gamma$ production by peripheral blood mononuclear cells (PBMCs) after stimulation with $C$. burnetii Phase 1 and Phase 2 antigen (Figure 2a).

More detailed analysis of the IFN- $\gamma$ Elispot results revealed a strong increase of the IFN- $\gamma$ producing PBMCs 


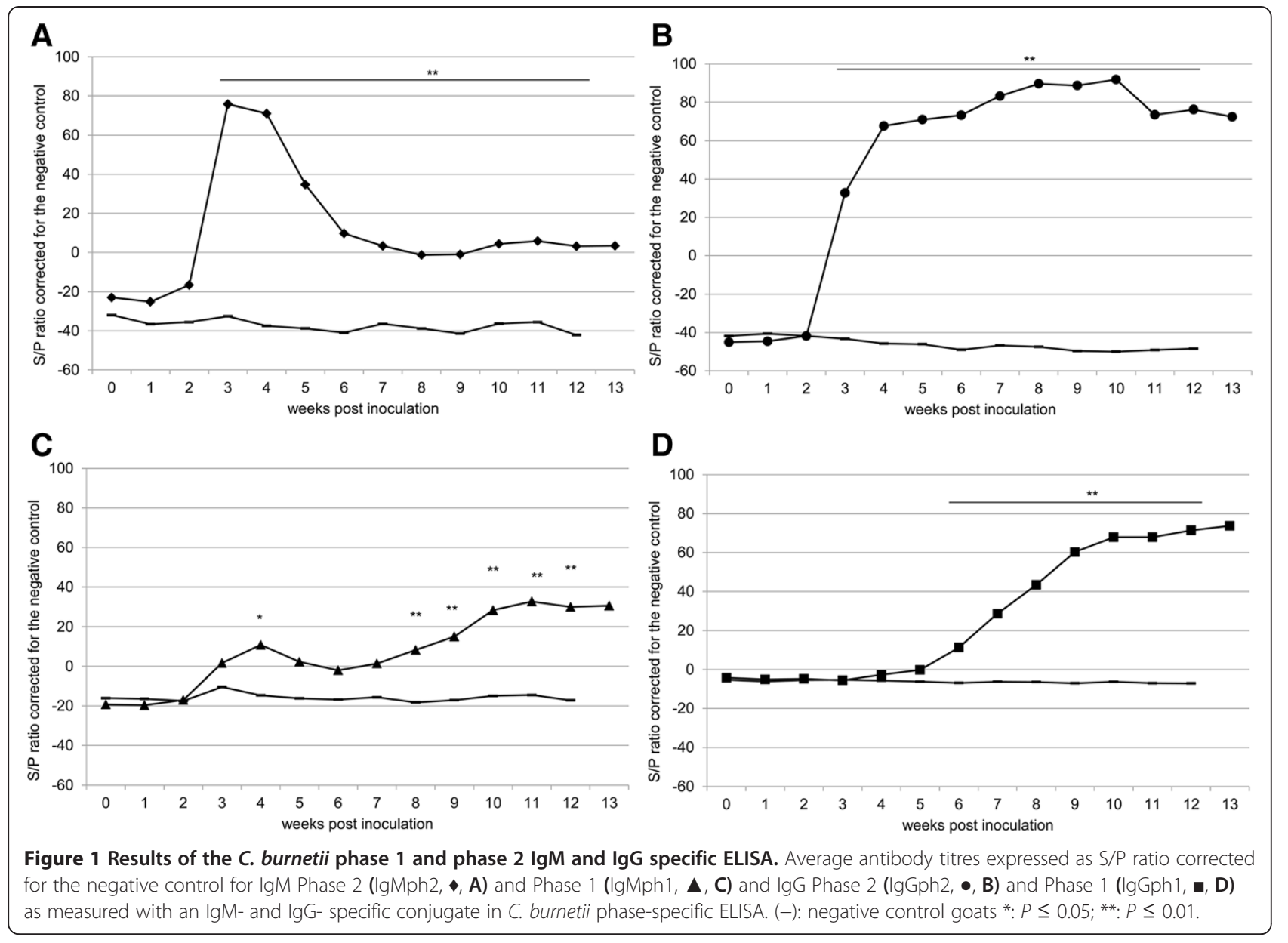

around the date of parturition for both Coxiella-inoculated goats and control goats. Upon correcting for the date of parturition, the increase in IFN-y production in both Coxiella-inoculated and control goats corresponded to the time of parturition (Figure $2 \mathrm{~b}$ ), suggesting that parturition influences IFN-y production in goats. In the further analysis of the IFN- $\gamma$ Elispot and LPT results, data were corrected for parturition. Stimulation with both Phase 1 and Phase 2 antigen resulted in a significant increase of the production of IFN- $\gamma$ one week after
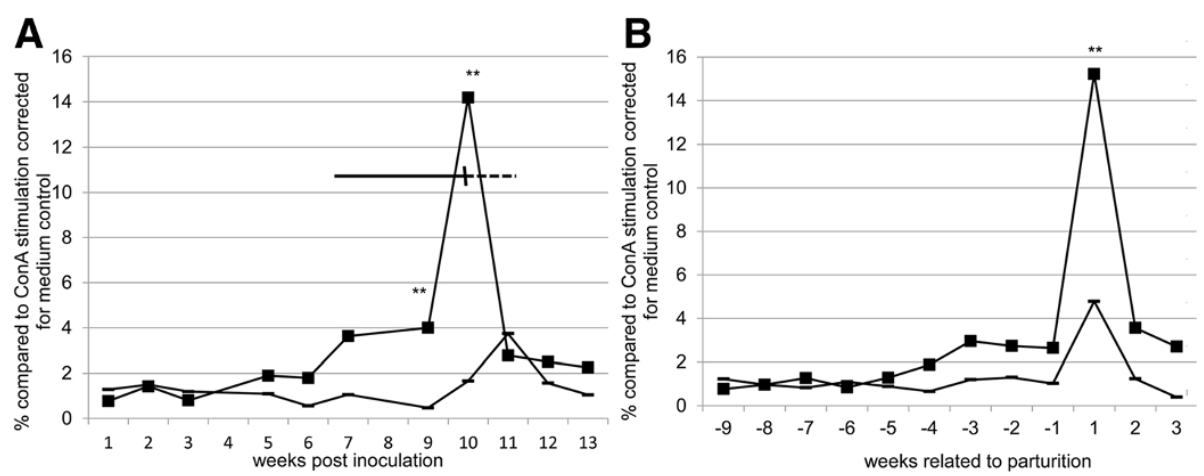

Figure 2 IFN-y Elispot results, related to inoculation and to parturition. Average data for PBMCs stimulated with Phase 1 and Phase 2 antigen. Data were corrected for medium-incubated cells and expressed as a percentage of ConA-stimulated cells. A: data were related to inoculation. Solid bar: delivery period of the Coxiella-inoculated goats; dotted bar: delivery period of the control goats. B: data related to delivery.

(-) Coxiella-inoculated goats; (-) negative control goats; *: $P \leq 0.05$; **: $P \leq 0.01$. 
parturition (Figure 3a and b). LPT results revealed an increase in proliferation ability of the PBMCs only after parturition in the Coxiella-inoculated goats (Figure 4a and b). The increase in proliferation ability after stimulation with $C$. burnetii Phase 2 antigen was stronger compared to stimulation with Phase 1 antigen (Figure $4 \mathrm{~b}$ ). Taken together, no clear cell-mediated immune response could be detected in the first weeks after infection. Only after parturition a significant increase in cell-mediated immune response was measured.

\section{Systemic cytokine mRNA responses}

Additional information about the cellular and humoral immune response was obtained via weekly measurement of systemic mRNA levels of the regulation of the proinflammatory cytokines TNF- $\alpha$ and IL- $1 \beta$ and the regulatory cytokines IL- 2 and IL-10. IFN- $\alpha$ and IFN- $\gamma$ mRNA regulation was measured likewise. Within 4 weeks after inoculation no differential effect on cytokine mRNA levels was measured between the Coxiella-inoculated group compared to the control group (data not shown). Systemic cytokine responses related to parturition are presented in Figure 5. The pro-inflammatory cytokines TNF- $\alpha$ and IL-1 $\beta$ mRNA were up regulated after parturition compared to the non-infected goats. No effect could be observed on IFN- $\alpha$ - and IFN- $\gamma$ mRNA levels. For the regulatory cytokines IL-2 and IL-10 mRNA, IL-2 was down regulated one week after parturition while IL10 was up regulated four and three weeks before parturition (Figure 5). Results indicate a significant systematic up regulation of TNF- $\alpha$, IL- $1 \beta$ and IL- 2 after parturition and a up regulation of IL-10 before parturition in the Coxiella-inoculated goats compared to the control goats.

\section{Discussion}

The goal of this experimental longitudinal study was to investigate the humoral and cellular immune responses in pregnant goats infected via a natural infection route with a Dutch outbreak isolate of $C$. burnetii. The immune response after $C$. burnetii inoculation via inhalation in domestic ruminants had not been studied yet. This inoculation route was successful in infecting goats and resulted in C. burnetii-infected placentas [14]. Up until now only subcutaneous inoculations had been used to study humoral immune responses [20,21]. However, the inoculation route might be important for studying naturally occurring immune responses.

Our results indicate a strong IgM anti-Phase 2 and IgG anti-Phase 2 humoral response, which starts to rise between 2 to 3 weeks post inoculation. This time period between inoculation and the first antibody response is shorter than reported for non-phase-specific antibodies in goats after subcutaneous inoculation [20,21], but is slightly longer than reported for humans and mice $[22,23]$. For goats, the difference with previous studies may be due to the use of different inoculation routes. Inoculation via inhalation can generate a mucosal immune response in the lungs; in humans, the lungs have the ability to respond quickly to some pathogens, i.e. Streptococcus pneumoniae. In the lungs, residential antigen-specific memory B cells are present but it is also reported that infections in the lung generate a de novo local and systemic antibody response [24]. In goats it is not known whether $C$. burnetii can induce such a response in the lung but the fast systemic antibody response could be a result of this.

The initial humoral immune response consisted of the generation of $C$. burnetii Phase 2-specific antibodies, whereas antibodies against $C$. burnetii Phase 1 arose at a later stage. Although in line with previously published work in mice, guinea pigs and humans [22,23,25], it is not clear why an anti-Phase 2 response is generated in advance of an anti-Phase 1 response, as Phase 1 Coxiella bacteria were inoculated. One possible explanation is
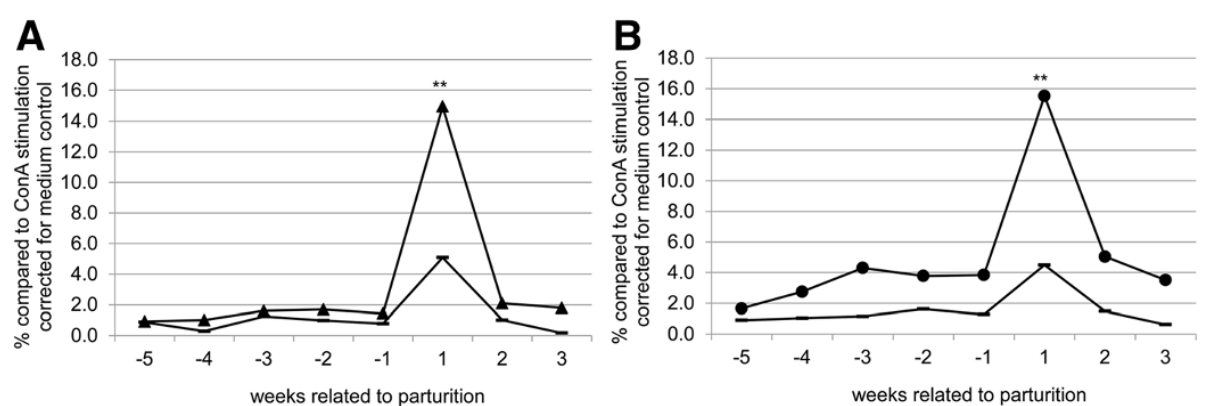

Figure 3 IFN-y Elispot results, per C. burnetii phase 1 and phase 2 stimulus, related to parturition. Results of the PBMC of control goats and Coxiella-inoculated goats after stimulation with C. burnetii Phase 1 or Phase 2 antigen. Data were corrected for medium-incubated cells and expressed as percentage of ConA-stimulated cells, the $\mathrm{X}$-axis is in weeks related to parturition (time point 0 ). $\mathbf{A}$ : results after stimulation with C. burnetii Phase 1 antigen, ( $\mathbf{\Lambda}$ : C. burnetii-inoculated goats; -: control goats). B: results after stimulation with C. burnetii Phase 2 antigen (•: C. burnetii-inoculated goats; - : control goats); ${ }^{*}: P \leq 0.05 ;{ }^{* *}: P \leq 0.01$. 

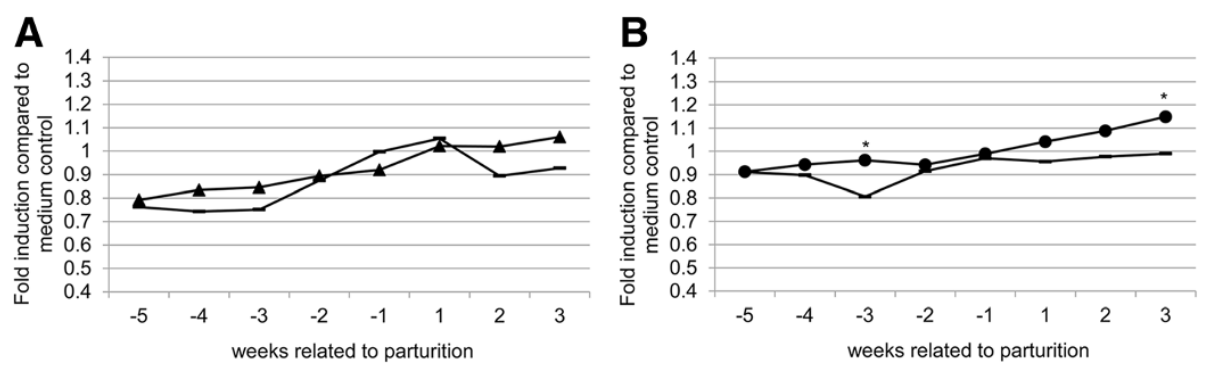

Figure 4 LPT results, per $C$. burnetii phase 1 and phase 2 stimulus, related to parturition. Lymphocyte proliferation test results of the control goats and Coxiella-inoculated goats. Data are expressed as average results of the group in fold induction compared to medium-incubated cells. The X-axis indicates weeks related to parturition (time point 0). A: results after stimulation with C. burnetii Phase 1 ( $\boldsymbol{\Lambda}$ : C. burnetii-inoculated goats; -: control goats). B: results after stimulation with C. burnetii Phase 2 antigen ( $\bullet$ : C. burnetii-inoculated goats; -: control goats); *: $P \leq 0.05$; **: $P \leq 0.01$

that besides Phase 1 bacteria, Phase 2 bacteria were inoculated as well. Phase 2 bacteria are efficiently internalised into phagosomes $[9,26]$. This results in an effective killing of the Phase 2 bacteria that gives rise to a humoral response against Phase 2 C. burnetii. Alternatively, it can be assumed that both LPS and surface protein antigens of Phase 1 C. burnetii are recognised by the immune system but that surface proteins, which Phase 1 and Phase 2 C. burnetii have in common [27], give an earlier and stronger humoral immune response compared to the LPS antigen. This results first in an anti-Phase 2 response to the surface antigens, followed by an anti-Phase 1 response against LPS. As we were not able to detect Phase 2 C. burnetii in our inoculum, we assume that both surface proteins and LPS are detected by the immune system. Therefore the initial anti-Phase 2 response may be due to an earlier and stronger response to surface proteins than to LPS.

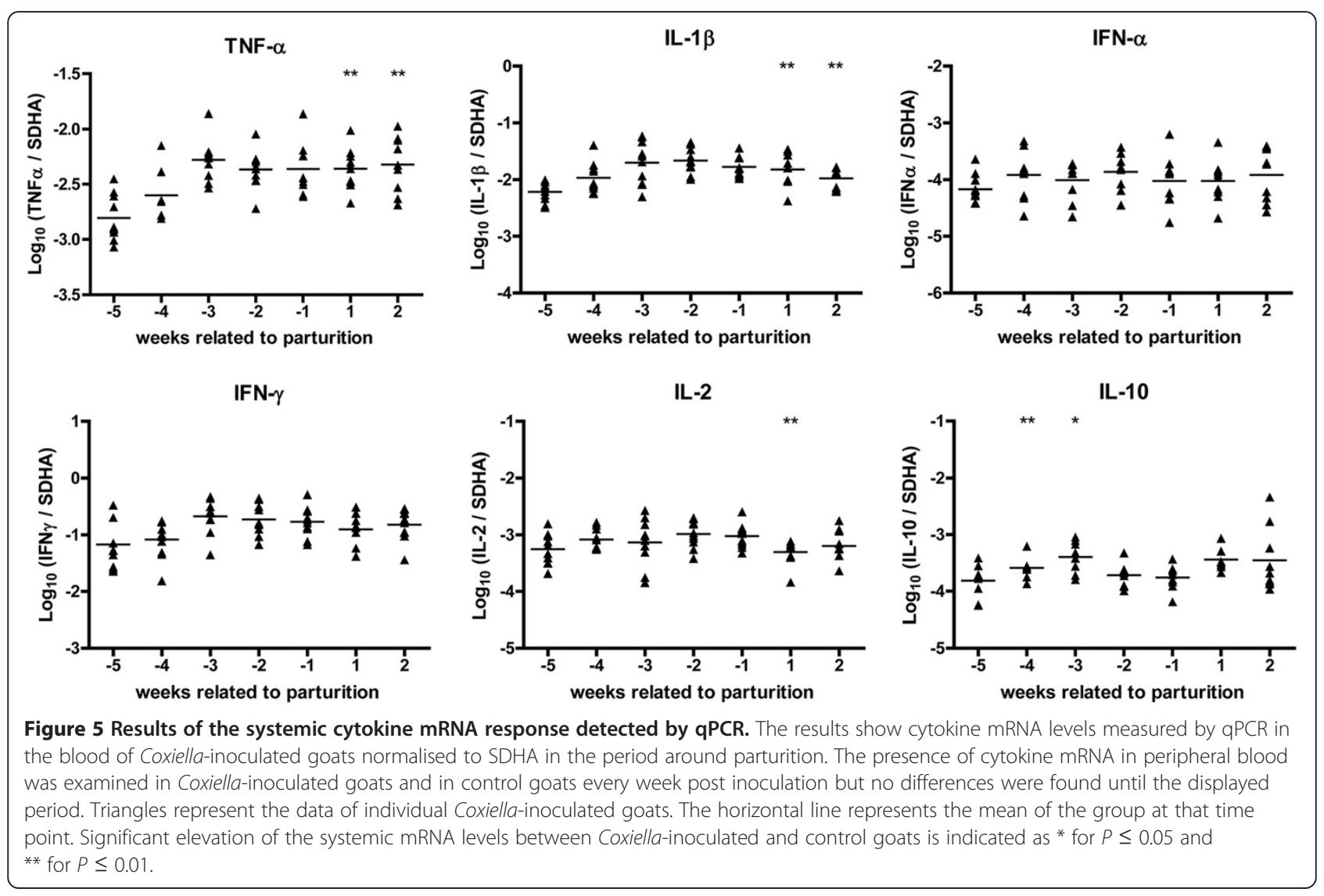


Surprisingly both anti-Phase 2 IgM and IgG titres started to rise at almost the same time. This was not expected, as IgM is generally the first immunoglobulin class to be produced in a humoral immune response because IgM can be expressed without class switching. During human Coxiella infection, IgM anti-Phase 2 antibody titres also start to rise first followed by a IgG antiPhase 2 response [22]. The phase-specific and antibodysubclass-specific humoral immune response might help in the detection of early or more prolonged C. burnetii infections in goats. As pregnant goats do not excrete the bacterium [14] the detection of anti-Phase $2 \operatorname{IgM}$ without anti-Phase 1 IgG will indicate an early infection. Whether phase-specific and antibody-subclass-specific antibody titres can predict or indicate chronic infections in goats should be investigated in further research, as the study period in the present study was only 13 weeks.

The cell-mediated immune response during the first weeks after inoculation was minimal, as indicated by the results of the IFN- $\gamma$ Elispot and the apparent absence of a systemic cytokine mRNA responses. This might indicate that the PBMCs have not been in contact with C. burnetii. This corresponds with our earlier results, which showed that Coxiella bacteria were not detectable in the blood after inoculation despite the infection of the trophoblasts of the placenta between 2 and 4 weeks after inoculation [14]. Studies in non-pregnant mice indicate that IFN- $\gamma$ has a role in the early control of $C$. burnetii proliferation [12]. This is probably not true in pregnant goats because we were not able to detect an increase in IFN- $\gamma$ producing cells in the weeks after inoculation. Therefore IFN- $\gamma$ probably does not play a role in preventing $C$. burnetii replication in the early stages of infection in pregnant goats.

Cell-mediated immune responses were first detected in the first week after parturition. At the time of parturition, the C. burnetii DNA load in the tissues is maximal as previously shown [14], resulting in exposure to the systemic immune system. This exposure coincides with an up regulation of the pro-inflammatory cytokines TNF- $\alpha$ and IL-1 $\beta$. This probably caused the strong increase in the total production of IFN- $\gamma$ by PBMCs at one week post parturition and the increase of the proliferation ability of PBMCs. However, parturition in noninfected goats also coincided with an increase in IFN- $\gamma$ producing PBMCs as measured in the control goats. This could be an effect of the release of suppression of the cell-mediated immune response after parturition. During pregnancy progesterone levels are high and decrease shortly before parturition [28]. In humans it is suggested that high progesterone levels are associated with a Th-2 type immunity, resulting in an increased humoral immune response and a decreased cell-mediated immune response [29]. In pregnant goats we also assume a down regulation of the cell-mediated immune response due to progesterone during pregnancy. If the suppression is alleviated after parturition, a strong increase in IFN- $\gamma$ producing cells can be expected. Overall, we assume that two mechanisms probably influence the cell-mediated immune response in Coxiella-infected pregnant goats, i.e. a stimulation by the release of Coxiella antigens and a suppression of cell-mediated immunity due to progesterone, with the overall effect that in infected goats no cell-mediated immune response was found during pregnancy.

IL-10 was up regulated towards the end of pregnancy in the Coxiella-inoculated goats. In mice as well as in humans persistent Coxiella presence seems to be IL-10 dependent $[9,30]$ and humans suffering from chronic Q fever excrete high levels of IL-10 [9]. It can be hypothised that Coxiella-infected pregnant goats may react similar as persistently infected mice and humans and reach a chronic infection status which is terminated by the induction of parturition. However, little is known about persistence of Coxiella infections in goats and how to detect these animals. In our earlier experiments on the pathogenesis of $\mathrm{Q}$ fever in pregnant goats we were not able to detect $C$. burnetii DNA at 81 days after parturition [14] although others were able to detect C. burnetii DNA in the genital tract of non-pregnant goats [31] and field observations may indicate long lasting shedding of $C$. burnetii in previously infected nonpregnant dairy goats [32]. To elucidate this further research is needed.

The results of our study may have several implications for diagnostic applications of immunological tests for $\mathrm{Q}$ fever in pregnant animals. Cell-mediated immune responses did not differ enough to distinguish between Coxiella-infected and non-infected pregnant goats. IFN- $\gamma$ Elispot, LPT and an additional performed IFN- $\gamma$ ELISA (data not shown, as results were over all even less discriminative) have no additional value in the diagnosis of $\mathrm{Q}$ fever in pregnant goats. The strong humoral response, however, is useful in the early detection of infected pregnant goats, because these goats cannot be diagnosed by the detection of $C$. burnetii [14]. The phase-specific IgM and IgG response might be useful in understanding the dynamics of $Q$ fever in a herd, as animals in different stages of infection can be followed.

\section{Competing interests}

The authors declare that they have no competing interests.

\section{Authors' contributions}

Conceived and designed the experiments: HIJR, JP, EvG, FGVZ, JMJR. Performed the experiments: HIJR, JP, EvG. Analyzed the data: HIJR, JP, EvG, JMJR. Wrote the paper: HIJR, JP, EvG, FGVZ, JMJR. All authors read and approved the final manuscript. 


\section{Acknowledgements}

The authors thank Frederique Bouvier and Francis Barillet from INRA (France) for their good cooperation in providing the goats. Colleagues from the department of Animal Technology are thanked for their dedicated care of the goats and sampling during the experiment, Arie Hoogendoorn, Lucien van Keulen and Annemieke Dinkla are thanked for technical assistance, Dimitrios Frangoulidis for providing the monoclonal C. burnetii antibody, Jeanet van der Goot for assistance with the statistics and Dave Thomas for editing the manuscript.

\section{Author details}

'Department of Bacteriology and TSEs, Central Veterinary Institute, part of Wageningen University and Research Centre, Edelhertweg 15, 8219, PH Lelystad, The Netherlands. ${ }^{2}$ Department of Infection Biology, Central Veterinary Institute, part of Wageningen University and Research Centre, Edelhertweg 15, 8219, PH Lelystad, the Netherlands.

Received: 19 December 2012 Accepted: 2 July 2013

Published: 1 August 2013

\section{References}

1. Greenslade E, Beasley R, Jennings L, Woodward A, Weinstein P: Has Coxiella burnetii ( $Q$ fever) been introduced into New Zealand? Emerg Infect Dis 2003, 9:138-140.

2. Babudieri B: Q fever: a zoonosis. Adv Vet Sci 1959, 5:81-154

3. Kersh GJ, Lambourn DM, Self JS, Akmajian AM, Stanton JB, Baszler TV, Raverty SA, Massung RF: Coxiella burnetii infection of a Steller sea lion (Eumetopias jubatus) found in Washington State. J Clin Microbiol 2010, 48:3428-3431

4. Roest $H I$, Hogerwerf $L$, van der Brom $R$, Oomen $T$, van Steenbergen JE, Nielen M, Vellema P: Q fever in the Netherlands: current status, results from veterinary research and expectations of the coming years. Tijdsch Diergeneeskd 2011, 136:340-343 (in Dutch).

5. Roest HI, Tilburg JJ, van der Hoek W, Vellema P, van Zijderveld FG, Klaassen $\mathrm{CH}$, Raoult D: The Q fever epidemic in The Netherlands: history, onset, response and reflection. Epidemiol Infect 2011, 139:1-12.

6. Kampschreur LM, Hagenaars JC, Wielders CC, Elsman P, Lestrade PJ, Koning $\mathrm{OH}$, Oosterheert JJ, Renders NH, Wever PC: Screening for Coxiella burnetii seroprevalence in chronic $Q$ fever high-risk groups reveals the magnitude of the Dutch Q fever outbreak. Epidemiol Infect 2013, 141:847-851.

7. Van Steenbergen JE, Morroy G, Groot CA, Ruikes FG, Marcelis JH, Speelman $P$ : An outbreak of $Q$ fever in The Netherlands-possible link to goats. Ned Tijdschr Geneeskd 2007, 151:1998-2003 (in Dutch).

8. Tilburg JJ, Rossen JW, van Hannen EJ, Melchers WJ, Hermans MH, van de Bovenkamp J, Roest HJ, de Bruin A, Nabuurs-Franssen MH, Horrevorts AM, Klaassen CH: Genotypic diversity of Coxiella burnetii in the 2007-2010 Q fever outbreak episodes in The Netherlands. J Clin Microbiol 2012, 50:1076-1078

9. Raoult D, Marrie TJ, Mege JL: Natural history and pathophysiology of $\mathrm{Q}$ fever. Lancet Infect Dis 2005, 5:219-226.

10. Toman R, Skultety L, Ihnatko R: Coxiella burnetii glycomics and proteomics-tools for linking structure to function. Ann N Y Acad Sci 2009, 1166:67-78

11. Moos A, Hackstadt T: Comparative virulence of intra- and interstrain lipopolysaccharide variants of Coxiella burnetii in the guinea pig model. Infect Immun 1987, 55:1144-1150.

12. Andoh M, Zhang G, Russell-Lodrigue KE, Shive HR, Weeks BR, Samuel JE: T cells are essential for bacterial clearance, and gamma interferon, tumor necrosis factor alpha, and B cells are crucial for disease development in Coxiella burnetii infection in mice. Infect Immun 2007, 75:3245-3255.

13. Maurin M, Raoult D: Q fever. Clin Microbiol Rev 1999, 12:518-553.

14. Roest HJ, van Gelderen B, Dinkla A, Frangoulidis D, van Zijderveld FG, Rebel $J$, van Keulen $L: Q$ fever in pregnant goats: pathogenesis and excretion of Coxiella burnetii. PLoS One 2012, 7:e48949.

15. Bottcher J, Vossen A, Janowetz B, Alex M, Gangl A, Randt A, Meier N: Insights into the dynamics of endemic Coxiella burnetii infection in cattle by application of phase-specific ELISAs in an infected dairy herd. Vet Microbiol 2011, 151:291-300.

16. Wang $Y$, Xiong $X$, Wu D, Wang $X$, Wen B: Efficient activation of T cells by human monocyte-derived dendritic cells (HMDCs) pulsed with Coxiella burnetii outer membrane protein Com1 but not by HspB-pulsed HMDCs. BMC Immunol 2011, 12:52.

17. Limonard GJ, Thijsen SF, Bossink AW, Asscheman A, Bouwman JJ: Developing a new clinical tool for diagnosing chronic $\mathrm{Q}$ fever: the Coxiella ELISPOT. FEMS Immunol Med Microbiol 2012, 64:57-60.

18. Zhang G, Samuel JE: Vaccines against Coxiella infection. Expert Rev Vaccines 2004, 3:577-584.

19. Rodolakis A, Clement P, Cochonneau D, Beaudeau F, Sarradin P, Guatteo R: Investigation of humoral and cellular immunity of dairy cattle after one or two year of vaccination with a phase I Coxiella vaccine. Procedia Vaccinol 2009, 1:85-88

20. Arricau Bouvery N, Souriau A, Lechopier P, Rodolakis A: Experimental Coxiella burnetii infection in pregnant goats: excretion routes. Vet Res 2003, 34:423-433.

21. Arricau-Bouvery N, Souriau A, Bodier C, Dufour P, Rousset E, Rodolakis A: Effect of vaccination with phase I and phase II Coxiella burnetii vaccines in pregnant goats. Vaccine 2005, 23:4392-4402.

22. Dupuis $\mathrm{G}$, Peter $\mathrm{O}$, Peacock M, Burgdorfer W, Haller E: Immunoglobulin responses in acute Q fever. J Clin Microbiol 1985, 22:484-487.

23. Kishimoto RA, Rozmiarek H, Larson EW: Experimental $Q$ fever infection in congenitally athymic nude mice. Infect Immun 1978, 22:69-71.

24. Twigg HL 3rd: Humoral immune defense (antibodies): recent advances. Proc Am Thorac Soc 2005, 2:417-421.

25. Williams JC, Damrow TA, Waag DM, Amano K: Characterization of a phase I Coxiella burnetii chloroform-methanol residue vaccine that induces active immunity against $\mathrm{Q}$ fever in $\mathrm{C} 57 \mathrm{BL} / 10 \mathrm{ScN}$ mice. Infect Immun 1986, 51:851-858.

26. Capo C, Lindberg FP, Meconi S, Zaffran Y, Tardei G, Brown EJ, Raoult D, Mege JL: Subversion of monocyte functions by Coxiella burnetii: impairment of the cross-talk between alphavbeta3 integrin and CR3. $\mathrm{J}$ Immunol 1999, 163:6078-6085.

27. Hackstadt T: Steric hindrance of antibody binding to surface proteins of Coxiella burnetii by phase I lipopolysaccharide. Infect Immun 1988, 56:802-807

28. Currie WB, Thorburn GD: Parturition in goats: studies on the interactions between the foetus, placenta, prostaglandin $\mathrm{F}$ and progesterone before parturition, at term or at parturition induced prematurely by corticotrophin infusion of the foetus. J Endocrinol 1977, 73:263-278.

29. Druckmann R, Druckmann MA: Progesterone and the immunology of pregnancy. J Steroid Biochem Mol B 2005, 97:389-396.

30. Meghari S, Bechah Y, Capo C, Lepidi H, Raoult D, Murray PJ, Mege JL: Persistent Coxiella burnetii infection in mice overexpressing IL-10: an efficient model for chronic Q fever pathogenesis. PLoS Pathog 2008, 4:e23.

31. Alsaleh A, Pellerin JL, Rodolakis A, Larrat M, Cochonneau D, Bruyas JF, Fien F: Detection of Coxiella burnetii, the agent of $\mathrm{Q}$ fever, in oviducts and uterine flushing media and in genital tract tissues of the non pregnant goat. Comp Immunol Microbiol Infect Dis 2011, 34:355-360.

32. Van den Brom R, Van E, Vos J, Luttikholt SJM, Moll L, Roest HIJ, van der Heijden HMJF, Vellema P: Detection of Coxiella burnetii in the bulk tank milk from a farm with vaccinated goats, by using a specific PCR technique. Small Rumin Res 2013, 110:150-154.

doi:10.1186/1297-9716-44-67

Cite this article as: Roest et al:: $\mathrm{Q}$ fever in pregnant goats: humoral and cellular immune responses. Veterinary Research 2013 44:67. 